\title{
Avaliação da insuficiência da veia safena magna com classificação C2 e C3 (CEAP) pela pletismografia a ar e pelo eco-Doppler colorido
}

\author{
Evaluation of the greater saphenous vein with classification C2 e C3 (CEAP) by air \\ plethysmography and color Doppler ultrasonography
}

Amélia Cristina Seidel', Leandro Pablos Rossetti², Yara Juliano 3 , Neil Ferreira Novo ${ }^{3}$, Fausto Miranda $\mathrm{r}^{4}$

\begin{abstract}
Resumo
Contexto: Com a presença de refluxo venoso, há necessidade de avaliar a gravidade clínica da doença pela quantificação do efeito hemodinâmico da incompetência venosa e definição de sua distribuição anatômica.

Objetivo: Determinar a correlação da pletismografia a ar com o grau de refluxo pelo eco-Doppler na insuficiência da veia safena magna no quadro clínico C2 e C3 da CEAP.

Métodos: Foram examinados, prospectivamente, 87 membros com refluxo da veia safena magna determinado pelo eco-Doppler e 32 membros sem sinais ou sintomas de doença venosa. Todos foram submetidos ao exame clínico, pletismografia e eco-Doppler de membros inferiores. Do ecoDoppler foram utilizados os parâmetros: diâmetro da veia safena em sete níveis, velocidade e tempo de refluxo. Da pletismografia foram considerados o índice de enchimento venoso, a fração de ejeção e a fração de volume residual.

Resultados: Dos 119 membros, 61 pertenciam à classe C2. Na comparação do diâmetro da veia nos grupos controle e estudo houve diferença estatisticamente significante, exceto ao nível do maléolo. Utilizando-se a Correlação de Spearman para análise dos índices da pletismografia e ecoDoppler foram observadas algumas significâncias, porém o coeficiente de explicação $\left(r^{2}\right)$ mostrou que foram fracas.

Conclusões: Os parâmetros da pletismografia não se correlacionam com o grau de refluxo na veia safena magna, pois houve uma correlação muito fraca entre seus valores e o tempo e a velocidade do refluxo. Somente o índice de enchimento venoso tem correlação com refluxo venoso. A fração de ejeção e de volume residual não se mostraram importantes na discriminação da gravidade clínica.
\end{abstract}

Palavras-chave: veia safena; ultrassonografia Doppler; pletismografia; varizes.

\begin{abstract}
Background: With the presence of venous reflux, there is need evaluate the clinical severity by quantifying the hemodynamic effect of venous incompetence and definition of their anatomical distribution.

Objective: To determine and correlate the degree of reflux of the greater saphenous vein (insufficiency) in a clinical CEAP C2/C3 by air plethysmography and color Doppler ultrasonography.

Methods: We prospectively investigated 87 limbs with reflux of the greater saphenous vein as ascertained by Doppler ultrasound and 32 limbs without signs or symptoms of the venous disease. All patients underwent clinical examinations using air plethysmography and Doppler ultrasound of the lower limbs. The parameters used with the Doppler ultrasound were: the diameter of the saphenous vein (seven levels) and the speed and time of reflux. In the plethysmography, the venous filling index, ejection fraction and residual volume fraction were also considered.

Results: Of the 119 limbs, 61 were class C2. In comparing the diameters of the vein of the control group with the study group there were statistically
\end{abstract}

Trabalho realizado na Disciplina de Angiologia e Cirurgia Vascular do Curso de Medicina da Universidade Estadual de Maringá (UEM) - Maringá (PR), Brasil, e no curso de Pós-Graduação da Universidade Federal de Paulo (UNIFESP-EPM) - São Paulo (SP), Brasil.

Trabalho apresentado no VIII Panamerican Congress on Vascular and Endovascular Surgery, I Panamerican Venous Forum, V Symposium on Vascular Echo-color-Doppler, em 2004; e no $15^{\text {th }}$ World Congress of the Union Internationale de Phebologie, em 2005. Classificado em segundo lugar do prêmio Peter Gloviczki.

${ }^{1}$ Ultrassonografista vascular pela Sociedade Brasileira de Angiologia e Cirurgia Vascular (SBACV) e pelo Colégio Brasileiro de Radiologia e Diagnóstico por Imagem (CBR); Professora Adjunta da Disciplina de Angiologia e Cirurgia Vascular do Curso de Medicina da UEM - Maringá (PR), Brasil.

${ }^{2}$ Acadêmico do curso de Medicina da UEM - Maringá (PR), Brasil.

${ }^{3}$ Professor Adjunto da Disciplina de Bioestatística do Departamento de Medicina Preventiva da UNIFESP-EPM e Professor Titular do Departamento de Saúde Pública da Universidade de Santo Amaro (UNISA) - São Paulo (SP), Brasil.

${ }^{4}$ Ultrassonografista vascular pela SBACV e CBR; Professor Titular da Disciplina de Cirurgia Vascular do Departamento de Cirurgia da UNIFESP-EPM - São Paulo (SP), Brasil.

Fonte de financiamento: nenhuma.

Conflitos de interesses: nada a declarar.

Submetido em: 26.07.11. Aceito em: 15.03.12.

J Vasc Bras. 2012;11(3):187-193. 
significant differences. There was an exception at the malleolus level. Using the Spearman correlation to analyze the indices for the plethysmography and Doppler ultrasound it showed some difference, but the coefficient of determination $\left(r^{2}\right)$ showed that they were weak.

Conclusions: The parameters of the plethysmography did not correlate with the degree of reflux in the greater saphenous vein. There was a very weak correlation between their values, time and speed of reflux. Only the venous filling index correlated with venous reflux. The ejection fraction and residual volume fraction were not important for discrimination of clinical severity.

Keywords: saphenous vein; Ultrasonography, Doppler; plethysmography; varicose veins.

\section{Introdução}

A insuficiência venosa crônica é uma doença muito prevalente na prática médica ${ }^{1,2}$. É caracterizada por um conjunto de alterações que podem atingir a pele e o tecido celular subcutâneo, determinada por insuficiência valvular e/ou falha da bomba muscular da panturrilha ${ }^{3}$. Essa doença apresenta consequências socioeconômicas significantes, pois suas complicações podem causar morbidades importantes ${ }^{4}$. Há vários fatores de risco associados ao desenvolvimento da insuficiência venosa crônica, como obesidade, sexo e idade ${ }^{5}$.

Veias varicosas representam somente parte do espectro da doença venosa, a qual engloba desde incompetência valvular assintomática até úlcera de perna, na maioria dos casos mais graves ${ }^{6}$. O refluxo é uma das maiores causas da doença, mas a quantidade mínima deste, que pode produzir sintomas, não é conhecida ${ }^{7}$.

A presença do refluxo venoso requer avaliação da gravidade clínica da doença, quantificação do efeito hemodinâmico da incompetência venosa e definição de sua distribuição anatômica ${ }^{8}$.

O eco-Doppler colorido permite uma detecção acurada e quantificação do refluxo em veias individuais ${ }^{8}$, e a introdução da pletismografia a ar (PGA) tem assegurado uma avaliação quantitativa da gravidade da insuficiência venosa crônica (IVC) não-invasivamente ${ }^{6,9,10}$.

Este trabalho visou investigar o valor da PGA no diagnóstico da insuficiência da veia safena magna (VSM) e determinar sua correlação com a distribuição anatômica do refluxo determinada pelo eco-Doppler colorido nos casos C2 e C3 (CEAP).

\section{Método}

Em um período de quatro meses, 352 pacientes que apresentavam queixas compatíveis com doença venosa foram atendidos em um laboratório vascular. Após avaliação clínica, foram submetidos aos exames de eco-Doppler colorido e PGA de membros inferiores. Entre estes, 71 apresentavam insuficiência de VSM nas classes clínicas C2 e C3.
Os participantes da pesquisa assinaram o termo de consentimento e o protocolo foi aprovado pelo Comitê de Ética em Pesquisa ( $\left.{ }^{\circ} 0832 / 02\right)$.

Este estudo é prospectivo transversal. Para inclusão no grupo E (estudo), foram considerados pacientes sem história de operação venosa, trombose venosa profunda ou flebite, e excluídos aqueles que apresentavam limitação na mobilização do tornozelo, joelho ou qualquer outra alteração musculoesquelética que pudesse interferir no esvaziamento venoso da panturrilha durante os exercícios na realização da PGA. Assim, o grupo foi formado com 87 membros de 56 pacientes do sexo feminino e 15 do sexo masculino com idade entre 24 e 78 anos.

Obedecendo aos mesmos critérios de inclusão e exclusão do grupo E e sem história atual de insuficiência venosa, para o grupo $\mathrm{C}$ (controle) foram convidados 16 indivíduos, sendo 14 do sexo feminino e 2 do sexo masculino, com idade entre 21 e 57 anos.

A classificação $\mathrm{CEAP}^{11}$ foi utilizada para avaliação clínica da doença venosa. No grupo E, os pacientes apresentavam apenas refluxo de etiologia primária no sistema superficial, sendo classificados como $\mathrm{C}_{2-3} \mathrm{EpAs}_{2-3}$ Pr. Os indivíduos do grupo $\mathrm{C}$ foram classificados como $\mathrm{C}_{0}$.

Foi utilizado um aparelho (Hewlett-Packard Image Point - Hewlett-Packard Co, Andover, Mass, USA) de eco-Doppler colorido para confirmar a perviedade do sistema venoso profundo dos membros inferiores dos pacientes e controles em decúbito dorsal. Em seguida, eles foram examinados em pé para avaliação das veias perfurantes, do diâmetro da VSM e da presença ou não do refluxo nas veias superficiais. A medida do diâmetro da VSM foi realizada em sete níveis diferentes, sendo que o primeiro foi considerado ainda na região inguinal, $2 \mathrm{~cm}$ distal à junção safeno-femoral, onde a veia era visibilizada sem qualquer curvatura. $\mathrm{O}$ quarto nível foi marcado na face medial do joelho, na altura da linha interarticular; o segundo e o terceiro localizados na coxa, equidistantes aos citados. O sétimo nível foi junto ao maléolo tibial medial e o quinto e o sexto localizados na perna, também equidistantes aos já citados.

Foi empregada a classificação do padrão de refluxo na VSM descrita por Engelhorn et al. ${ }^{12}$ caracterizada por tipo: 
I - com refluxo perijunção; II - com refluxo em segmento proximal; III - com refluxo em segmento distal; IV - com refluxo segmentar; V - com refluxo de distribuição difusa. $\mathrm{Na}$ análise espectral, o fluxo retrógrado presente na VSM foi definido como refluxo com duração maior que 0,5 segundo ${ }^{13}$ e velocidade sistólica maior que $25 \mathrm{~cm} / \mathrm{s}$.

Em outra sala, com temperatura controlada entre $22 \mathrm{e} 24^{\circ} \mathrm{C}$, foi realizada a PGA (aparelho SDV 3000 marca Angiotec ${ }^{\circledR}$ ) baseada no método descrito por Christopoulos et al. ${ }^{14}$, com registro do índice de enchimento venoso (VFI: venous filling index), da fração de ejeção (EF: ejection fraction) e da fração de volume residual (RVF: residual volume fraction) para avaliação hemodinâmica do membro. Foram considerados valores normais o $\mathrm{VFI}<2 \mathrm{~mL} / \mathrm{s}, \mathrm{EF}>60 \%$ e $\mathrm{RVF}<40 \%$.

$\mathrm{Na}$ análise estatística, foi utilizado o teste $t$ de Student para dados independentes, avaliando a homogeneidade da amostra e a correlação de Spearman para verificação dos índices obtidos na PGA e no eco-Doppler colorido. Referese ao valor do coeficiente como zero quando duas variáveis são absolutamente independentes entre si, ou seja, não existe nenhuma correlação entre elas, e é considerado valor máximo de +1 quando a associação for positiva e o mais "forte" possível, e valor máximo de -1 quando for negativa e mais "fraca". A correlação é significante com nível de 5\%.

\section{Resultados}

Os indivíduos dos grupos $\mathrm{C}$ e $\mathrm{E}$ foram avaliados quanto ao peso, altura e índice de massa corporal pelo teste $t$ de Student para dados independentes que confirmaram a homogeneidade da amostra (Tabela 1).

Todos do grupo C, de acordo com a classificação CEAP, pertenciam à classe clínica $\mathrm{C}_{0}$ e não apresentavam refluxo da VSM. Já os pacientes do grupo $\mathrm{E}$, das classes clínicas $\mathrm{C}_{2}$ e $\mathrm{C}_{3}$, pelo eco-Doppler colorido, foram distribuídos quanto ao tipo de refluxo na VSM (Tabela 2).

Pela correlação de Spearman, na comparação do diâmetro da VSM com os valores dos índices obtidos na PGA, observou-se correlação significante entre o VFI e as medidas do diâmetro da VSM em alguns níveis nos grupos $\mathrm{C}$ e E, mas de maneira muito fraca. Com a EF e a RVF, a mesma correlação foi observada, mas somente no grupo E (Tabela 3).

Com o mesmo teste estatístico, na análise do diâmetro da VSM com o tempo do refluxo e o pico de velocidade do refluxo, as correlações mantiveram-se fracas em alguns níveis nos diferentes padrões de refluxo (Tabela 4).

Na comparação do pico da velocidade do refluxo ao Doppler com os valores dos índices da PGA (VFI, EF e
Tabela 1. Teste $t$ de Student para avaliação da homogeneidade da amostra quanto ao peso, altura e índice de massa corporal.

\begin{tabular}{lcc}
\hline \multicolumn{3}{c}{ Grupo Ex Grupo C $\left(\mathrm{t}_{\text {crit }}=1,96\right)$} \\
\hline Peso & $\mathrm{t}_{\text {cal }}$ & Valor $\mathrm{p}$ \\
\hline Altura & 0,75 & 0,45 \\
Índice de massa corpora & 0,06 & 0,95 \\
\hline $\mathrm{t}_{\text {crit: }} \mathrm{t}$ crítico; $\mathrm{t}_{\text {cal }} \mathrm{t}$ calculado. & 0,75 & 0,45 \\
FSignificância $<5 \%$. & &
\end{tabular}

Tabela 2. Distribuição dos membros inferiores dos pacientes do grupo E das classes clínicas C2 e C3 segundo o tipo de refluxo na veia safena magna.

\begin{tabular}{lccc}
\hline \multicolumn{4}{c}{ Classes clínicas CEAP } \\
\hline $\begin{array}{l}\text { Tipo de refluxo ao eco- } \\
\text { Doppler colorido }\end{array}$ & 2 & 3 & Total \\
\hline II & 34 & 11 & 45 \\
III & 6 & - & 6 \\
IV & 15 & 3 & 18 \\
V & 6 & 12 & 18 \\
\hline Total & 61 & 26 & 87 \\
\hline
\end{tabular}

Tabela 3. Resultados da Correlação de Spearman na análise entre o diâmetro da veia safena magna e os parâmetros da pletismografia a ar nos grupos $\mathrm{C}$ e $\mathrm{E}$.

\begin{tabular}{|c|c|c|c|c|c|c|c|c|c|c|c|c|}
\hline \multirow{3}{*}{ Nível } & \multicolumn{4}{|c|}{ VFI } & \multirow{2}{*}{\multicolumn{2}{|c|}{$\frac{\text { EF }}{\text { Tipo II }}$}} & \multicolumn{6}{|c|}{ RVF } \\
\hline & \multicolumn{2}{|c|}{ Controle } & \multicolumn{2}{|c|}{ Tipo IV } & & & \multicolumn{2}{|c|}{ Tipo II } & \multicolumn{2}{|c|}{ Tipo III } & \multicolumn{2}{|c|}{ Tipo V } \\
\hline & $r$ & $\mathrm{P}^{*}$ & $r$ & $\mathrm{P}^{*}$ & $r$ & $\mathrm{P}^{*}$ & $r$ & $\mathrm{P}^{*}$ & $r$ & $\mathrm{P}^{*}$ & $r$ & $\mathrm{P}^{*}$ \\
\hline 1 & 0,3 & 0,0 & 0,0 & 0,7 & 0,4 & 0,0 & 0,5 & 0,0 & 0,2 & 0,8 & 0,0 & 0,9 \\
\hline 2 & 0,5 & 0,0 & 0,2 & 0,4 & $0,4^{* *}$ & $0,0^{* *}$ & 0,4 & 0,0 & 0,3 & 0,6 & 0,1 & 0,7 \\
\hline 3 & 0,5 & 0,0 & 0,0 & 0,9 & 0,3 & 0,0 & $0,3^{* *}$ & $0,0^{* *}$ & 0,0 & 1,0 & $0,5^{* *}$ & $0,0^{* *}$ \\
\hline 4 & 0,6 & 0,0 & 0,0 & 0,9 & 0,3 & 0,0 & 0,5 & 0,0 & $0,9^{* *}$ & $0,0^{* *}$ & 0,4 & 0,0 \\
\hline 5 & $0,4^{* *}$ & $0,0^{* *}$ & $0,5^{* *}$ & $0,0^{* *}$ & 0,2 & 0,3 & 0,2 & 0,1 & 0,6 & 0,3 & 0,4 & 0,0 \\
\hline 6 & 0,3 & 0,1 & $0,5^{* *}$ & $0,0^{* *}$ & 0,0 & 0,8 & 0,0 & 1,0 & 0,6 & 0,3 & 0,1 & 0,6 \\
\hline 7 & 0,4 & 0,0 & 0,3 & 0,2 & 0,0 & 0,6 & 0,2 & 0,2 & 0,0 & 1,0 & 0,2 & 0,4 \\
\hline
\end{tabular}

VFI: venousfillingindex; EF: ejectionfraction; RVF: residual volumefraction; r: coeficiente de correlação.

*Significância <5\%; **Variáveis significativas.

Tabela 4. Resultados da Correlação de Spearman na análise entre o diâmetro da veia safena magna, o tempo de refluxo e o pico da velocidade de refluxo no grupo $E$.

\begin{tabular}{|c|c|c|c|c|c|c|}
\hline \multirow{3}{*}{$\begin{array}{c}\text { Diâmetro } \\
\text { Nível }\end{array}$} & \multicolumn{4}{|c|}{ Tempo de refluxo } & \multirow{2}{*}{\multicolumn{2}{|c|}{$\frac{\text { Pico de velocidade de refluxo }}{\text { Tipo IV }}$}} \\
\hline & \multicolumn{2}{|c|}{ Tipo III } & \multicolumn{2}{|c|}{ Tipo IV } & & \\
\hline & $r$ & $\mathrm{P}^{*}$ & $r$ & $\mathrm{P}^{*}$ & $r$ & $\mathrm{P}^{*}$ \\
\hline 1 & 0,77 & 0,77 & 0,28 & 0,26 & 0,08 & 0,75 \\
\hline 2 & $0,84^{* *}$ & $0,03^{* *}$ & 0,10 & 0,68 & 0,42 & 0,08 \\
\hline 3 & 0,51 & 0,30 & 0,30 & 0,24 & 0,07 & 0,77 \\
\hline 4 & 0,24 & 0,65 & $0,56^{* *}$ & $0,01^{* *}$ & 0,18 & 0,48 \\
\hline 5 & 0,04 & 0,94 & 0,12 & 0,63 & 0,42 & 0,08 \\
\hline 6 & 0,22 & 0,68 & 0,18 & 0,48 & $0,53^{* *}$ & $0,02^{* *}$ \\
\hline 7 & 0,49 & 0,33 & 0,41 & 0,09 & 0,44 & 0,07 \\
\hline
\end{tabular}

r: coeficiente de correlação.

*Significância <5\%; *Variáveis significativas. 
RVF) dos pacientes do grupo E houve diferença estatisticamente significante, com correlação muito fraca no VFI dos padrões de refluxo II e IV e da RVF do padrão II, inferindose que pode haver VSM com refluxo e não existir alteração no valor do VFI (Tabela 5, Figuras 1 e 2).

Quando correlacionados o tempo do refluxo venoso e os valores obtidos na PGA, nos diferentes padrões de refluxo, não foi obtida diferença significante.

No grupo $C$ não houve veias perfurantes insuficientes. No grupo E foi mais comum a presença de uma perfurante insuficiente em cada membro, em todos os grupos, com prevalência de $46,7 \%$ no tipo II, $50,0 \%$ no tipo III, $36,8 \%$ no tipo IV e $33,3 \%$ no tipo V.

Todos os índices obtidos (VFI, EF e RVF, tempo e pico da velocidade de refluxo) nos exames realizados (PGA e eco-Doppler colorido) também foram correlacionados estatisticamente com os valores dos diâmetros das veias perfurantes insuficientes presentes nos membros de cada paciente do grupo E. Na comparação do diâmetro destas veias com o VFI houve correlação significante no tipo de refluxo $\mathrm{V}$, com r 0,46; e EF e RVF no tipo II com coeficiente 0,32 e 0,28 , respectivamente. Quando comparados com a velocidade do refluxo, houve correlação significante nos tipos II e V com r 0,27 e 0,53, respectivamente (Tabela 6). Não houve significância na comparação com o tempo de refluxo. Estes resultados demonstraram fraca correlação estatística, podendo-se inferir que o diâmetro das veias perfurantes insuficientes também teve pouca influência nos resultados da PGA.

\section{Discussão}

Para avaliar a importância da PGA na quantificação da insuficiência da VSM foi realizada análise comparativa desta com a avaliação clínica do paciente e o eco-Doppler colorido dos membros inferiores.

O estudo foi dirigido para as classes $\mathrm{C} 2$ e C 3 da CEAP por serem os pacientes mais frequentemente encaminhados para tratamento de varizes dos membros inferiores, isto é, aqueles com doença do sistema venoso superficial e perfurantes insuficientes que apresentam varizes, sem outros sinais ou sintomas de complicações.

Neste trabalho, na distribuição da classificação de refluxo, predominou o tipo II (55\%), seguido dos tipos V (20\%), IV (19\%) e III (6\%). Engelhorn et al. ${ }^{12}$ encontraram como o mais comum o tipo IV $(38,4 \%)$, seguido dos tipos II $(13,8 \%)$, III $(7,4 \%), \mathrm{V}(6,6 \%)$ e I $(0,8 \%)$. Por sua vez, Luccas et al. ${ }^{15}$ obtiveram $45,2 \%$ dos pacientes com VSM normal, $13,6 \%$ com insuficiência em toda a extensão da VSM, 17,0\% com insuficiência da crossa até a região do joelho, 11,1\% com
Tabela 5. Resultados da Correlação de Spearman na análise entre a velocidade de refluxo e os parâmetros da pletismografia a ar no grupo $E$, em que o coeficiente demonstrou correlação.

\begin{tabular}{|c|c|c|c|c|c|c|}
\hline \multirow{3}{*}{ Velocidade } & \multicolumn{4}{|c|}{ VFI } & \multirow{2}{*}{\multicolumn{2}{|c|}{$\frac{\text { RVF }}{\text { Tipo II }}$}} \\
\hline & \multicolumn{2}{|c|}{ Tipo II } & \multicolumn{2}{|c|}{ Tipo IV } & & \\
\hline & $r$ & $\mathrm{P}^{*}$ & $r$ & $P^{*}$ & $r$ & $\mathrm{P}^{*}$ \\
\hline$>25 \mathrm{~cm} / \mathrm{s}$ & 0,37 & 0,01 & 0,52 & 0,02 & 0,32 & 0,03 \\
\hline
\end{tabular}

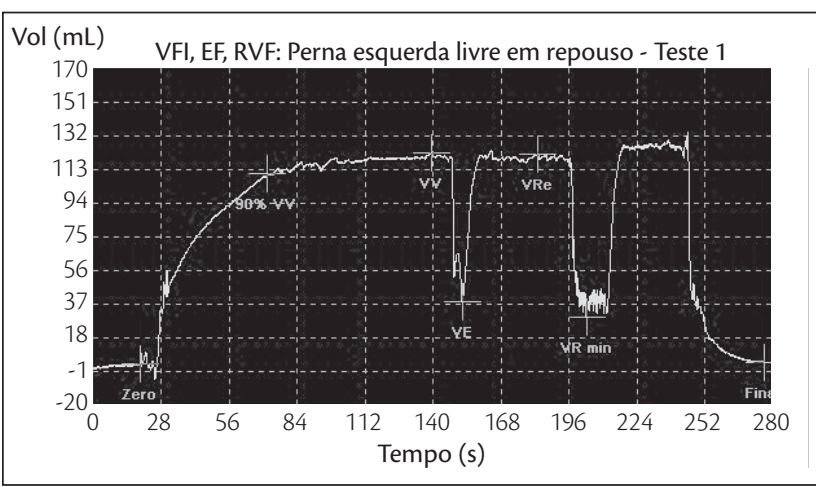

VFI: índice de enchimento venoso; EF: fração de ejeção; RVF: fração de volume residual. Figura 1. Fotografia do gráfico obtido como resultado da pletismografia a ar em paciente com padrão de refluxo da veia safena magna tipo $\mathrm{V}$ com índice de enchimento venoso de $1,73 \mathrm{~mL} / \mathrm{s}$ (normal).

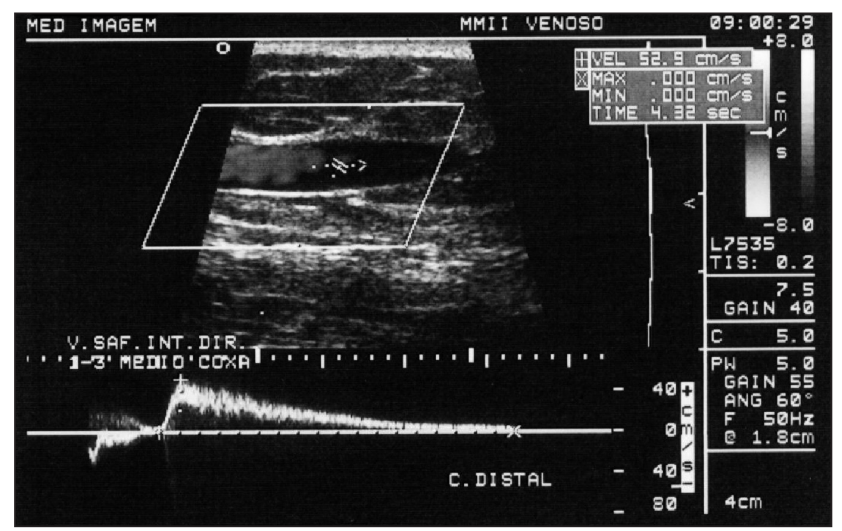

Figura 2. Fotografia do resultado do eco-Doppler colorido em paciente com refluxo da veia safena magna tipo $V$ com velocidade de $52,9 \mathrm{~cm} / \mathrm{s}$ e tempo 4,32 s. Mesmo paciente da Figura 1.

Tabela 6. Resultado da Correlação de Spearman entre os diâmetros das veias perfurantes insuficientes, os parâmetros da pletismografia a ar, o tempo e a velocidade de refluxo nos diferentes padrões ultrassonográficos de refluxo da veia safena magna.

\begin{tabular}{lccccc}
\hline \multicolumn{5}{c}{ Diâmetro das perfurantes } \\
\hline \multirow{2}{*}{ Variável } & \multicolumn{3}{c}{$\mathrm{II}$} & & \multicolumn{2}{c}{$\mathrm{V}$} \\
\cline { 2 - 3 } \cline { 5 - 6 } & $\mathrm{r}$ & $\mathrm{P}^{*}$ & & $\mathrm{r}$ & $\mathrm{P}^{*}$ \\
\hline VFI & 0,38 & 0,00 & & $0,46^{* *}$ & $0,04^{* *}$ \\
EF & $0,32^{* *}$ & $0,02^{* *}$ & & 0,04 & 0,87 \\
RVF & $0,28^{* *}$ & $0,05^{* *}$ & & 0,00 & 0,99 \\
Tempo & 0,22 & 0,10 & & 0,11 & 0,63 \\
Velocidade & $0,27^{* *}$ & $0,05^{* *}$ & & $0,53^{* *}$ & $0,02^{* *}$ \\
\hline
\end{tabular}

r: coeficiente de correlação; VFI: índice de enchimento venoso; EF: fração de ejeção; RVF: fração de volume residual.

*Significância $<5 \%$; **Variáveis significativas. 
insuficiência da crossa ao $1 / 3$ superior ou médio da coxa, $3,4 \%$ com insuficiência apenas da crossa e de suas colaterais e 9,4\% com padrão insuficiência segmentar entre $1 / 3$ médio da coxa e 1/3 superior da perna. Acreditamos que a diferença dos valores encontrados seja devida ao tipo de paciente que cada serviço recebe para exame.

Considerando que o exame clínico pode ser falho na determinação da fonte do refluxo, o eco-Doppler colorido é, hoje, reconhecido como método de escolha para seu diagnóstico em veias individuais ${ }^{13,16-21}$.

A literatura não ofereceu parâmetros comparativos para discutir o pico da velocidade do refluxo no segmento insuficiente da VSM, pois com a medida da velocidade em vários segmentos do sistema venoso há dificuldade no cálculo do índice de refluxo total e, também, a multiplicidade das veias e os erros inerentes a este cálculo fazem tal medida difícil e de pouca credibilidade ${ }^{22}$.

$\mathrm{Na}$ análise da PGA, os índices pletismográficos (VFI, EF) são um método razoável para distinguir a presença ou ausência de insuficiência venosa crônica ${ }^{23,24}$, mas não discriminam bem membros com veias varicosas não complicadas de membros com úlceras ou dermatite de estase, e o VFI correlaciona-se pobremente com a presença de veias incompetentes e seus diâmetros ${ }^{25}$.

A EF pode não apresentar correlação com o grau clínico da doença. A RVF, o VFI e o tempo de fechamento valvular têm demonstrado tendência à piora da hemodinâmica venosa se a IVC progride, com significante sobreposição entre os grupos em todos os parâmetros testados, exceto quando comparados o grupo controle e aqueles com doença venosa ${ }^{26}$.

Teoricamente, o VFI e a eficácia da bomba muscular da panturrilha (EF) deveriam correlacionar-se com manifestações da IVC. Entretanto, no presente trabalho esta correlação não foi observada.

Christopoulos et al. ${ }^{14}$ encontraram RVF de 2 a $35 \%$ em membros normais e 35 a $65 \%$ em membros com veias varicosas. No presente trabalho, a média dos valores da RVF foi $28,2 \%$ para membros normais e $32,0,26,0,30,4$ e $36,1 \%$ nos tipos II, III, IV e V de refluxo da VSM, respectivamente, havendo nítida sobreposição dos valores encontrados.

As informações funcionais fornecidas nas diversas fases do teste descrito são importantes para a avaliação clínica e decisão terapêutica ${ }^{27,28}$, mas estudando a variação dos parâmetros da PGA na doença venosa crônica em situações de teste-reteste, os autores concluíram que é pouco provável que a PGA detecte pequenas alterações nos parâmetros de avaliação do refluxo venoso e da função da bomba muscular da panturrilha, exceto se a amostra estudada for grande $^{29}$. Há trabalhos que concluíram que a PGA não é um teste sensível o suficiente para detectar pequenas alterações hemodinâmicas ${ }^{21,25,26,30,31}$, mas o VFI é um parâmetro diagnóstico útil na avaliação da insuficiência venosa crônica ${ }^{23,32}$.

Resultados obtidos relacionando os índices da PGA e eco-Doppler colorido demonstraram que o VFI correlaciona-se significantemente com o tempo total do refluxo do membro e o tempo de refluxo no sistema venoso profundo, concluindo que as informações obtidas por estes dois exames parecem ser complementares por fornecerem dados hemodinâmicos e informações anatômicas detalhadas do segmento estudado ${ }^{33,34}$.

Diferente do trabalho citado, outros autores não encontraram correlação entre o tempo de fechamento valvular e o volume do fluxo, ou entre o tempo de fechamento valvular e o pico da velocidade do refluxo em nenhuma localização anatômica estudada. Também nenhuma correlação foi encontrada entre tempo de fechamento valvular e o VFI, ou entre o volume total do fluxo e a RVF ${ }^{35}$.

A correlação entre o VFI e o pico da velocidade do refluxo só foi observada nos pacientes com padrão de refluxo tipo II e IV, sendo de fraca intensidade com coeficientes iguais a 0,37 e 0,52 , respectivamente.

Para estudo de pacientes nas diferentes classes clínicas, é recomendado o emprego do eco-Doppler colorido e PGA para uma completa avaliação do membro com $\mathrm{IVC}^{22}$, mas van Bemmelen et al. ${ }^{25}$ citaram que os parâmetros da PGA não distinguiram eficientemente membros com IVC daqueles com veias varicosas não complicadas. Sugeriram que a discrepância entre os exames seria porque não mediram o mesmo refluxo, ocorrendo o efeito do fluxo arterial na medida da PGA.

Este trabalho apresenta resultados semelhantes ao realizado por Yamaki et al. ${ }^{22}$, o qual concluiu que uma duração aumentada do refluxo não se correlacionou com o VFI. Entretanto, há significante correlação entre o VFI e o pico da velocidade na junção safeno-femoral e safeno-poplítea, e moderada correlação com refluxo na VSM. A EF e RVF têm somente pobre correlação com parâmetros derivados do eco-Doppler colorido. A duração do refluxo em veias individuais mostrou ter fraca correlação com parâmetros da PGA e a gravidade clínica CEAP em pacientes com insuficiência venosa superficial isolada.

Ocorre piora no quadro clínico da insuficiência venosa dependente do número e o diâmetro das veias perfurantes insuficientes ${ }^{36,37}$, mas segundo Labropoulos et al. ${ }^{36}$, outros fatores devem ser considerados e, portanto, é difícil provar esta associação.

Na amostra estudada, os resultados estatísticos também apresentaram correlação muito fraca entre o diâmetro das 
veias perfurantes, os índices da PGA (somente EF e RVF) e a velocidade do refluxo nos padrões de refluxo da VSM tipos II e V.

Os dados da literatura indicam que o eco-Doppler colorido é mais acurado em avaliar o sistema venoso profundo e superficial isoladamente, mas a adição da PGA ajuda a quantificar o grau da incompetência superficial, identifica obstrução ao fluxo e ajuda a determinar se varicosidades são importantes contribuintes para elevar a pressão venosa ou são canais de escoamento necessários de veias profundas obstruídas $22,25,38$.

\section{Conclusão}

A PGA não se mostrou útil na avaliação da insuficiência da veia safena magna nos quadros clínicos C2 e C3 (CEAP).

\section{Referências}

1. França LHG, Tavares V. Insuficiência venosa crônica: uma atualização. J Vasc Bras. 2003;2(4):318-28.

2. Castro e Silva M, Cabral ALS, Barros Jr N, Castro AA, Santos MERC Diagnóstico e tratamento da doença venosa crônica. J Vasc Bras. 2005;4(3):S185-94.

3. Vasdekis SN, Clarke GH, Nicolaides AN. Quantification of venous reflux by means of duplex scanning. J Vasc Surg. 1989;10(6):670-7. http://dx.doi.org/10.1016/0741-5214(89)90011-6

4. Ting AC, Cheng SW, Wu LL, Cheung GC. Air plethysmography in chronic venous insufficiency: clinical diagnosis and quantitative assessment. Angiology. 1999;50(10):831-6. PMid:10535722.

5. Seidel AC, Mangolim AS, Rossetti LP, Gomes JR, Miranda Jr F. Prevalência de insuficiência venosa superficial dos membros inferiores em pacientes obesos e não obesos. J Vasc Bras. 2011;10(2):124-30. http://dx.doi.org/10.1590/ S1677-54492011000200006

6. Antignani PL. Classification of chronic venous insufficiency: a review. Angiology. 2001;52(Suppl 1):S17-26.

7. Christopoulos D, Nicolaides AN, Szendro G. Venous reflux: quantification and correlation with the clinical severity of chronic venous disease. Br J Surg. 1988;75(4):352-6. PMid:3359149.

8. Criado E, Farber MA, Marston WA, Daniel PF, Burnham CB, Keagy $B A$. The role of air plethysmography in the diagnosis of chronic venous insufficiency. J Vasc Surg. 1998;27(4):660-70. http://dx.doi. org/10.1016/S0741-5214(98)70231-9

9. Dezotti NRA, Joviliano EE, Toma MK, Moriya T, Piccinato CE. Estudo da hemodinâmica venosa por meio da pletismografia a ar no pré e pós-operatório de varizes dos membros inferiores. J Vasc Bras. 2009;8(1):21-8.

10. Saliba Jr OA, Giannini M, Rollo HA. Métodos de diagnóstico nãoinvasivos para avaliação da insuficiência venosa dos membros inferiores. J Vasc Bras. 2007;6(3):266-75.
11. Eklöf B, Rutherford RB, Bergan JJ, Carpentier PH, Gloviczki P, Kistner $\mathrm{RL}$, et al. Revision of the CEAP classification for chronic venous disorders: consensus statement. J Vasc Surg. 2004;40(6):1248-52. http://dx.doi.org/10.1016/j.jvs.2004.09.027

12. Engelhorn CA, Picheth FS, Castro Jr N, Dabul Jr NM, Gomes CS. Estudo do sistema venoso superficial com o duplex scan a cores. Cir Vasc Angiol. 1996;12:12-4.

13. van Bemmelen PS, Bedford G, Beach K, Strandness DE. Quantitative segmental evaluation of venous valvular reflux with duplex ultrasound scanning. J Vasc Surg. 1989;10(4):425-31. http://dx.doi. org/10.1016/0741-5214(89)90417-5

14. Christopoulos DG, Nicolaides AN, Szendro G, Irvine AT, Bull $M L$, Eastcott $\mathrm{HH}$. Air-plethysmography and the effect of elastic compression on venous hemodynamics of the leg. I Vasc Surg. 1987;5(1):148-59. http://dx.doi.org/10.1016/0741-5214(87)90205-9

15. Luccas GC, Nagase Y, Menezes FH, Matsui IA, Capri LG, Silveira SAF, et al. Cirurgia de varizes dos membros inferiores. Avaliação pré-operatória do sistema venoso com mapeamento duplex. Cir Vasc Angiol. 1996;12:15-20.

16. Cavezzi A, Labropoulos N, Partsch H, Ricci S, Gaggiati A, Myers K, et al. Duplex ultrasound investigation of the veins in chronic venous disease of the lower limbs - UIP consensus document. Part II. Anatomy. Eur J Vasc Endovasc Surg. 2006;31(3):288-99.

17. Labropoulos N, Leon LR Jr. Duplex evalution of venous insufficiency. Semin Vasc Surg. 2005;18(1):5-9. http://dx.doi.org/10.1053/j. semvascsurg.2004.12.002

18. Delis KT, Ibegbuna V, Nicolaides AN, Lauro A, Hafez H. Prevalence and distribution of incompetent perforating veins in chronic venous insufficiency. J Vasc Surg. 1998;28(5):815-25. http://dx.doi. org/10.1016/S0741-5214(98)70056-4

19. Szendro G, Nicolaides AN, Zukowski AJ, Christopoulos D, Malouf GM, Christodoulou C, et al. Duplex scanning in the assessment of deep venous incompetence. J Vasc Surg. 1986;4(3):237-42. http:// dx.doi.org/10.1016/0741-5214(86)90192-8

20. Neglén P, Raju S. A rational approach to detection of significant reflux with duplex Doppler scanning and air plethysmography. J Vasc Surg. 1993;17:590-5. http://dx.doi. org/10.1016/0741-5214(93)90160-N

21. Welch HJ, Faliakou EC, McLaughlin RL, Umphrey SE, Belkin M, O'Donnell TF Jr. Comparison of descending phlebography with quantitative photoplethysmography, air plethysmography, and duplex quantitative valve closure time in assessing deep venous reflux. J Vasc Surg. 1992;16(6):913-9. http://dx.doi. org/10.1016/0741-5214(92)90054-C

22. Yamaki T, Nozaki M, Sasaki K. Quantitative assessment of superficial venous insufficiency using duplex ultrasound and air plethysmography. Dermatol Surg. 2000;26(7):644-8. http://dx.doi. org/10.1046/j.1524-4725.2000.00025.x

23. Nishibe T, Kudo F, Miyazaki K, Kondo Y, Nishibe M, Dardik A. Relationship between air-plethysmographic venous function and clinical severity in primary varicose veins. Int Angiol. 2006;25(4):3525. PMid: 17164740

24. Oliveira RA, Barros Jr N, Miranda Jr F. A variabilidade hemodinâmica venosa detectada pelos parâmetros da pletismografia a ar nas classes clínicas da classificação CEAP. J Vasc Bras. 2007;6(4):359-65. 
25. van Bemmelen PS, Mattos MA, Hodgson KJ, Barkmeier LD, Ramsey DE, Faught WE, et al. Does air plethysmography correlate with duplex scanning in patients with chronic venous insufficiency? J Vasc Surg. 1993;18(5):796-807. http://dx.doi. org/10.1016/0741-5214(93)90334-I

26. lafrati MD, Welch $H$, O'Donnell TF, Belkin M, Umphrey $S$, McLaughlin R. Correlation of venous noninvasive tests with the Society for Vascular Surgery / International Society for Cardiovascular Surgery clinical classification of chronic venous insufficiency. J Vasc Surg. 1994;19(6):1001-7. http://dx.doi. org/10.1016/S0741-5214(94)70211-X

27. Comerota AJ, Harada RN, Eze AR, Katz ML. Air plethysmography: a clinical review. Int Angiol. 1995;14(1):45-52. PMid:7658103.

28. Evangelista SSM. Pletismografia no estudo das doenças venosas. In: Maffei FHA, Lastória S, Yoshida WB, Rollo HA, organizadores. Doenças vasculares periféricas. 3 ed. Rio de Janeiro: Medsi; 2002. p. 479-92.

29. Yang D, Vandongen YK, Stacey MC. Variability and reliability of air plethysmographic measurements for the evaluation of chronic venous disease. J Vasc Surg. 1997;26(4):638-42. http://dx.doi. org/10.1016/S0741-5214(97)70063-6

30. Payne SP, Thrush AJ, London NJ, Bell PR, Barrie WW. Venous assessment using air plethysmography: a comparison with clinical examination, ambulatory venous pressure measurement and duplex scanning. Br J Surg. 1993;80(8):967-70. http://dx.doi.org/10.1002/ bjs. 1800800808

31. Araki $\mathrm{CT}$, Back TL, Padberg FT Jr, Thompson PN, Duran WN, Hobson RW 2nd. Refinements in the ultrasonic detection of popliteal vein reflux. J Vasc Surg. 1993;18(5):742-8. http://dx.doi. org/10.1016/0741-5214(93)90326-H

32. Engelhorn CA, Beffa CV, Bochi G, Pullig RC, Cassou MF, Cunha SS A pletismografia a ar avalia a gravidade da insuficiência venosa crônica? J Vasc Bras. 2004;3:311-6.

33. Weingarten MS, Czeredarczuk M, Scovell S, Branas CC, Mignogna GM, Wolferth CC Jr. A correlation of air plethysmography and COlor-flow-assisted duplex scanning in the quantification of chronic venous insufficiency. J Vasc Surg. 1996;24(5):750-4. http://dx.doi. org/10.1016/S0741-5214(96)70008-3

34. Navarro TP, Delis KT, Ribeiro AP. Clinical and hemodynamic significance of the greater saphenous vein diameter in chronic venous insufficiency. Arch Surg. 2002;137(11):1233-7. PMid:12413308.

35. Rodriguez AA, Whitehead CM, McLaughlin RL, Umphrey SE, Welch HJ, O'Donnell TF. Duplex-derived valve closure times fail to correlate with reflux flow volumes in patients with chronic venous insufficiency. J Vasc Surg. 1996;23(4):606-10. http://dx.doi. org/10.1016/S0741-5214(96)80039-5

36. Labropoulos N, Tassiopoulos AK, Bhatti AF, Leon L. Development of reflux in the perfurator veins in limbs with primary venous disease. J Vasc Surg. 2006;43(3):558-62. http://dx.doi.org/10.1016/j. jvs.2005.11.046

37. Krnic A, Vucic N, Sucic Z. Correlation of perforating vein incompetence with extent of great saphenous insufficiency: cross sectional study. Croat Med J. 2005;46(2):245-51. PMid:15849846

38. Hanrahan LM, Araki CT, Fisher JB, Rodriguez AA, Walker TG, Woodson J, et al. Evaluation of the perforating veins of the lower extremity using high resolution duplex imaging. J Cardiovasc Surg. 1991;32(1):87-97. PMid:2010459.

Correspondência
Amélia Cristina Seidel
Rua Dr. Gerardo Braga, 118 - Jardim Vila Rica
CEP 87050-610 - Maringá (PR), Brasil
E-mail: seidel@wnet.com.br
Contribuições dos autores
Análise e interpretação dos dados: ACS, LPR, FMJ.
Coleta de dados: ACS.
Redação do artigo: ACS, LPR.
Revisão crítica do texto: ACS, LPR, FMJ.
Aprovação final do artigo*: ACS, FMJ.
Análise estatística: YJ, NFN.
Responsabilidade geral pelo estudo: ACS
* Todos os autores leram e aprovaram a versão final submetida ao J Vasc Bras.

